\title{
SUSTAINABILITY
}

\section{Environmental impact of food equity}

\author{
Nat. Sustain. https://doi.org/10.1038/s41893-019-0371-6
}

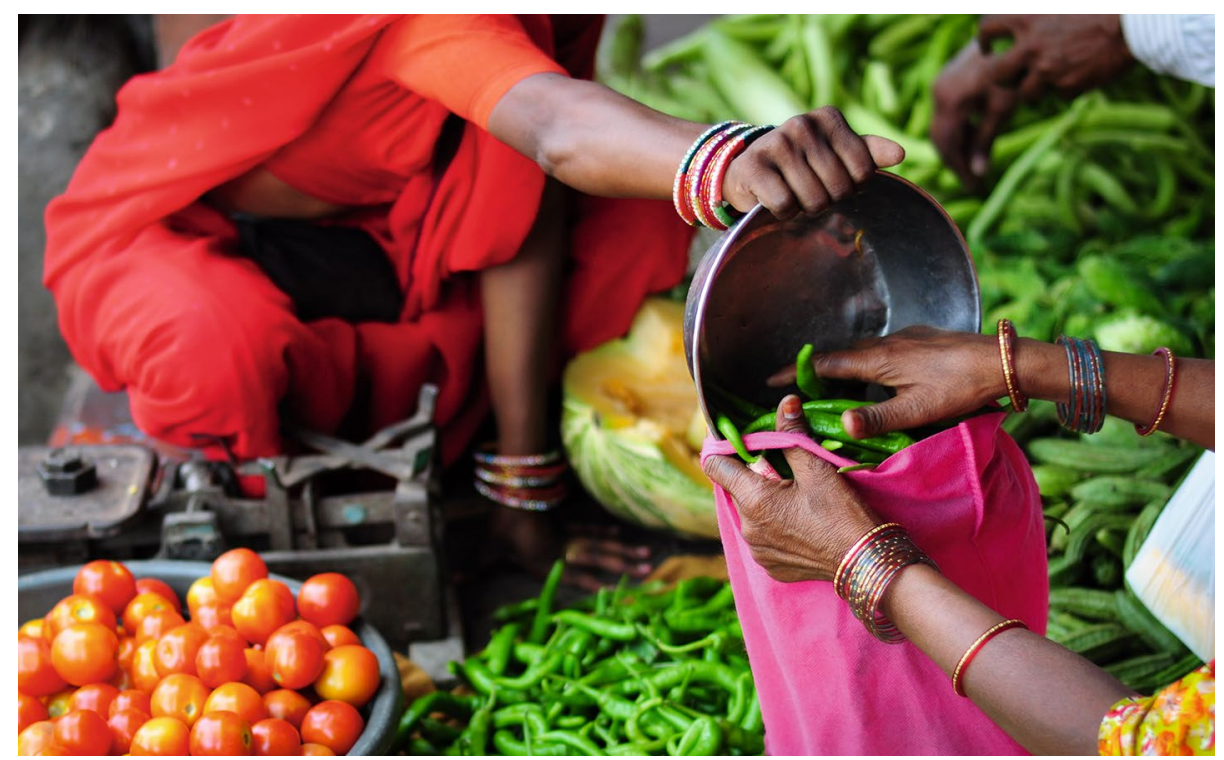

Credit: RAZVAN CIUCA / Moment / Getty

The global population is growing; how will our food system cope? This question is particularly important in light of the environmental damage that the global food system already inflicts on the planet.

In a new modelling study, Tomoko Hasegawa of Ritsumeikan University and colleagues asked whether solutions that focus on ensuring more food for the hungry, rather than more food for all, might differ in their environmental footprint. Their results show that increasing food production and availability for all will indeed require a sharp increase in food production of $20 \%$ by 2030 , requiring an additional 48 million hectares (MHa) of land for agriculture. However, targeting only those populations that are calorie-deficient will require just a $3 \%$ rise in production and under $15 \mathrm{MHa}$ of land in the same timeframe. Importantly, scenarios that build on this by also tackling overconsumption and waste could negate the need for increased food production entirely, resulting in land sparing.

These findings have implications for policymakers: initiatives that target foodinsecure groups, and address waste and overconsumption, are likely to have far lower environmental impacts than initiatives that aim to increase overall food availability via agricultural intensification. Increased food equity and reduced food waste can deliver a food-secure future for all.

\section{Charlotte Payne}

Published online: 23 October 2019

https://doi.org/10.1038/s41562-019-0773-5 